\title{
Epigenetics in the Central Nervous System
}

\author{
Kaili Anier • Anti Kalda
}

Published online: 2 September 2012

(C) Springer Science+Business Media, LLC 2012

\begin{abstract}
Emerging evidence suggests that epigenetic alterations to the genome, including DNA methylation, histone modifications, and microRNA synthesis are important mechanisms underlying several physiological and pathological processes in brain. In this review, we introduce the reader to epigenetic mechanisms and describe a potential role for dynamic epigenetic changes in memory formation, aging, and mediating addictive behaviors via long-lasting changes in gene expression. We also discuss evidence for a "histone code" in the central nervous system that mediates synaptic plasticity, learning, and memory. Collectively, the current rapid growth in epigenetic research could potentially revolutionize our understanding of the molecular changes associated with several neuropsychiatric diseases. Moreover, these studies demonstrate that alteration of the histone modification and/or the DNA methylation might prove to be potent candidates for therapeutic interventions.
\end{abstract}

Keywords Gene expression · Epigenetics · Chromatin structure · Histone modifications · DNA methylation · Non-coding RNA · Histone code · Memory formation · Aging $\cdot$ Drug addiction

\section{Introduction}

The sequence of nucleotides comprising an individual's genome is identical, with the exception of a few rare somatic mutations, across all cells in the body. However, DNA is structurally much more complex than a string of nucleotides, and at a functional level the genome is anything but

K. Anier $\cdot$ A. Kalda $(\bowtie)$

Department of Pharmacology, University of Tartu,

19 Ravila Street,

Tartu 50411, Estonia

e-mail: anti.kalda@ut.ee static. While every cell in our bodies contains the same DNA sequence, each has its own unique phenotype characterized by a specific pattern of gene expression that is in a constant state of flux. In the context of determining the phenotype of a cell, of additional importance is the degree to which specific genes are functionally active at any particular time in development. Therefore, sequencing the genome was only the first step in our quest to understand how genes are expressed and regulated. Accumulating evidence indicates that above the DNA sequence is a second layer of information - the epigenome - that regulates several genomic functions, including when and where genes are turned on or off. Historically, Conrad Hal Waddington coined the term epigenetics (literally meaning "above genetics") in 1942 to describe the examination of causal mechanisms whereby the genes of the genotype bring about phenotypic effects $[1,2]$. At the present time, epigenetics is defined as any heritable changes that affect gene expression without altering the DNA sequence itself [3, 4]. Epigenetic processes are essential for normal cellular development and differentiation, and allow the long-term regulation of gene function through non-mutagenic mechanisms. Increasing reports suggest that alterations of epigenetic mechanisms affect the vast majority of nuclear processes (including gene transcription and silencing), DNA replication and repair, cell cycle, telomere and centromere function and structure [5]. During the last decade, the field of epigenetics has rapidly developed into one of the most influential areas of scientific research and has become an important topic in several neurobiology fields such as learning and memory, psychiatric and neurological disorders.

The aim of this review is to introduce the potential role that epigenetic mechanisms play in physiological processes, such as memory formation and aging, and in the pathogenesis of complex diseases, such as drug addiction, mediating addictive behaviors by triggering long-lasting changes in gene expression in response to addictive substances. 


\section{Epigenetics Processes}

\section{Chromatin Structure}

Epigenetics is used to refer to the extremely complex processes of organizing the genome in a manner that allows for regulated gene expression in the appropriate cell type upon appropriate cellular stimuli. The fundamental unit that accomplishes this feat on a molecular level is chromatin, which is a complex of DNA, histones and non-histone proteins in the cell nucleus. The basic repeating structural unit of chromatin is a nucleosome (Fig. 1), which consists of $\sim 147$ base pairs (bp) of DNA wrapped around a core nucleosome [6॰]. Nucleosomes are composed of octamers that contain four histone homodimers, one each of histones H2A, H2B, H3 and $\mathrm{H} 4$, with $\mathrm{H} 1$ binding to spans of non-nucleosomal DNA. The histone-DNA configuration is maintained by electrostatic bonds between positively charged histones and negatively charged DNA [7]. This highly condensed histone proteins-DNA complex structure means that control over gene expression occurs partly by gating access of transcriptional activators to DNA $[8,9]$. Numerous types of posttranslational modifications of the amino-terminal tails of histones alter chromatin compaction to create more "open" states (euchromatin, which is transcriptionally permissive) versus "closed" states (heterochromatin, which is transcriptionally repressive) [10].

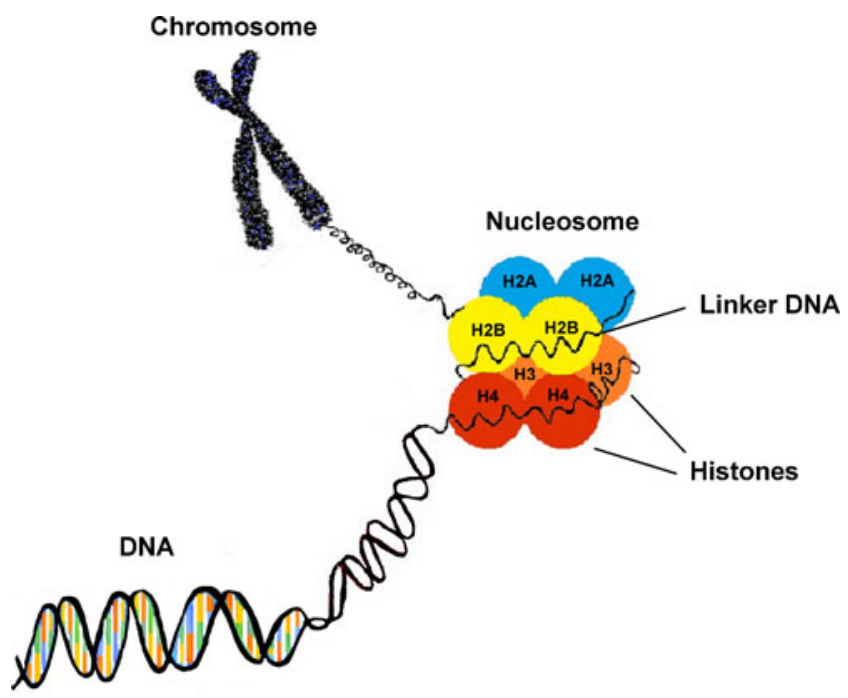

Fig. 1 DNA is wrapped around a cluster of histone proteins to form nucleosomes. The two main epigenetic modifications are associated with transcription. In histone modifications, a combination of different molecules can attach to the tails of histones, which consequently change the state of the chromatin around the DNA. When the chromatin becomes opened, the transcription of associated genes is activated and opposite when chromatin becomes closed. In DNA methylation, methyl marks added in $\mathrm{CpG}$ islands generally repress gene transcription (adapted from [88])
Histone Modifications

Each histone protein is composed of a central globular domain and an N-terminal tail that contains multiple sites for posttranslational modifications, including acetylation, phosphorylation, methylation, ubiquitination, and ADPribosylation. Most histone modifications are dynamic [11-13] and are regulated by a large number of histonemodifying enzymes like acetyltransferases, deacetylases, methyltransferases, demethylases, kinases, etc. [14].

The enzymes that regulate levels of histone acetylation are histone acetyltransferases (HAT's). The primary function of HAT's is to neutralize the charges on histones to relax chromatin structure, allowing for greater access to the DNA by transcription factors and therefore increase transcription $[15,16]$. On ther other hand, histone deacetylases (HDAC's) deacetylate histone tails and histone deacetylation have been linked to transcriptional repression [17-19]. Both HAT's and HDAC's comprise multiple enzyme classes whose expression and activity are exquisitely regulated [20]. The balance between the opposing activities of HAT's and HDAC's determines the level of expression of genes.

Another group of important enzymes are histone methyltransferases (HMT's), which are methylated at lysine or arginine residues and removed by histone demethylases (HDM's) [21-23]. Interestingly, a number of histone sites can undergo dimethylation or even tri-methylation [24, 25]. Histone methylation has been associated with both transcriptional activation and repression, depending on the particular residue and the extent of methylation [26, 27].

Phosphorylation of serine or threonine residues on histone tails can be accomplished by a broad range of nuclear kinases such has mitogen- and stress-activated protein kinase (MSK-1) and can be dephosphorylated by protein phosphatases (like protein phosphatase 1, PP1) $[28,29]$. The exact mechanism through which phosphorylation contributes to transcriptional activation is not well understood, but it is hypothesized that the addition of negatively charged phosphate groups to histone tails neutralizes the basic charge of histone tails and reduces their affinity for DNA [30].

\section{Histone Code}

Histone modifications are capable of being both genespecific within the genome and site-specific within a given chromatin particle, meaning that they are in an ideal position to selectively influence gene expression. While the genespecific modifications change transcription of specific genes, then site-specific modifications are known to directly alter chromatin state and transcription through a number of mechanisms. For example, histone acetylation, phosphorylation, and ubiquitination are generally associated with 
transcriptional activation, whereas histone methylation and other modifications (sumoylation, deimination, and proline isomerization) are more closely correlated with transcriptional repression [31,32]. Given that histone proteins can be modified at a number of sites raises the possibility that specific modifications could work together as a "code," which dictates whether a specific gene was transcribed. This "histone code" hypothesis suggests that certain combinations of histone modifications will lead to transcriptional activation, whereas others would lead to transcriptional repression $[6 \bullet, 18,33,34]$.

Yet another means by which specific histone modifications could combine to produce a unique epigenetic signature is via the inherent kinetics underlying each reaction [35]. Histone acetylation and phosphorylation are likely reversed very rapidly, whereas histone methylation may persist for longer periods of time. This would allow these mechanisms to synergistically control gene expression across unique time courses despite having no direct interactions.

\section{DNA Methylation}

DNA methylation is the second important epigenetic mechanism. The methylation of one of the four DNA bases, cytosine, is the most stable epigenetic modification, regulating the transcriptional plasticity of mammalian genomes. In DNA methylation, the methyl group is added to the 5, position on the cytosine pyrimidine ring and this occurs primarily where a cytosine $(C)$ occurs next to guanine $(G)$ in the DNA sequence $(\mathrm{C}$ - phosphate link - $\mathrm{G}$, or cytosineguanine dinucleotides, $\mathrm{CpG}$ ) [36, 37]. The $\mathrm{CpG}$ sequences are not evenly dispersed throughout the genome, but are clustered in so-called $\mathrm{CpG}$ islands - short regions of 0.5 to $4 \mathrm{~kb}$ in length having a rich (60-70\%) cytosine-guanine content. Over $50-60 \%$ of all dinucleotides in these islands are $\mathrm{CpG}$, compared to the rest of the genome where the $\mathrm{CpG}$ content is $\leq 20 \%$ [38]. About $50 \%$ of $\mathrm{CpG}$ islands are located in the promoter regions and around the transcription start sites and are unmethylated in normal cells. Proper DNA methylation is required for normal development, genetic imprinting and X-chromosomal inactivation [39].

DNA methylation is generally considered to repress gene transcription through recruitment of corepressor complexes (for example, HDAC's and HMT's) that can sterically hinder the transcriptional machinery or modify nucleosome structure. Such complexes involve several DNA methylbinding domain proteins, which are required for normal cell growth and development [39-41]. There is also evidence of DNA methylation in which gene transcription can have an activating role $[42,43 \bullet \cdot]$.

Cytosine methylation is catalysed by a family of enzymes termed DNA methyltransferases (DNMT's). In mammalian genomes, DNMT's are the only enzymes that have been shown to mediate the transfer of the methyl group from Sadenosylmethionine to cytosine [44-46]. There are two main enzyme groups: the DNMT1 and DNMT3 families. DNMT1 recognizes hemimethylated DNA, attaches a methyl group to the complementary cytosine base and is essential for maintaining DNA methylation patterns in proliferating cells [46-48]. The DNMT3 family includes two active de novo DNA methyltransferases-DNMT3A and DNMT3Band they are necessary for establishing new DNA methylation patterns $[46,47]$. Currently, there is no consensus about DNA demethylases in mammalian cells, although several potential canditates have been proposed [49-51].

\section{Non-coding RNA}

Another level of epigenetic regulation by small non-coding RNAs (termed microRNAs) has been discovered [52]. MicroRNAs, which are generally around 22 bp long, are important in biological regulation and are found in all mammalian cells [53]. MicroRNAs are posttranslational regulators that bind to complementary sequences on target mRNAs and regulate gene expression at different levels, i.e., the silencing of chromatin (affecting histone modifications), degradation of mRNA and blocking translation [53-55]. Thus, like histone modifications and DNA methylation, microRNAs can also be considered important players in the epigenetic control of gene expression.

\section{Epigenetic Mechanisms in Memory Formation}

Accumulating evidence suggests that epigenetic mechanisms, like histone modifications and DNA methylation, play an essential role in transcriptional regulation, synaptic plasticity and memory formation $[16,56]$. For example, a contextual fear conditioning (a hippocampus-dependent form of memory) coincides with increased acetylation, methylation, and/or phosphorylation at multiple sites of histone $\mathrm{H} 3$ and $\mathrm{H} 4$ tails in the rodent hippocampus CA1 region $[57,58 \cdot]$. None of these changes occur in control animals (exposed to the same context without fear conditioning), therefore these data indicate that such modifications are specific to associative learning.

In addition, several studies have demonstrated that interference with the molecular machinery that regulates histone modifications (acetylation, phosphorylation, methylation) disrupts associative learning and long-term potentiation (LTP). Specifically, histone acetylation upregulation with sodium butyrate (known as HDAC inhibitor) enhances memory formation and LTP [59]. Korzus and colleagues reported that genetic disruption of HAT activity impairs hippocampal memory and these deficits are rescued by 
HDAC inhibitor trichostatin A (TSA) [60]. Likewise, local administration of HDAC inhibitor TSA into the mouse hippocampus improves spatial long-term memory [61]. Recently, it has been shown that mice with deletion of HDAC2 demonstrated increased fear conditioning and hippocampal LTP, while HDAC2 overexpression in the mouse hippocampus blunts LTP and impairs memory [62・•]. Furthermore, it has been found that HDAC2 was enriched at several gene promoters like Bdnf, Fos, Creb and Zif268, and may inhibit the expression of these memory associated genes by binding to their regulatory elements [63]. Similarly for histone acetylation, mice with genetic deletion of specific histone methyltransferase displayed deficits in contextual fear conditioning, suggesting that histone methylation is required for proper long-term consolidation of contextual fear memories [58•]. Taken together, these data suggest that multiple histone modifications work together as a "histone code," and in the context of learning and memory this code produces unique changes in gene expression required for memory formation.

Mounting evidence indicates that changes in DNA methylation also represent a critical molecular component of both the formation and maintenance of long-term memories [63, $64,65 \bullet \cdot, 66]$. It has been found that contextual fear conditioning consequently increases and decreases DNA methylation of memory-related genes expressed in the hippocampus and these changes are necessary for memory formation. For example, contextual fear conditioning leads to an increase in the expression of DNMT3A and DNMT3B mRNA in the rat hippocampal area CA1, and inhibition of DNMT's within the hippocampus, which produces a hypomethylated state in naive animals, resulting in impaired expression of contextual fear memories $[64,66]$. In addition, the fear conditioning caused rapid but reversible methylation of the memory suppressor $P P 1$ and demethylation of the reelin (a gene involved in synaptic plasticity and memory). Increased $P P 1$ methylation was associated with $P P 1$ transcriptional silencing and reelin demethylation was associated with transcriptional activation. Changes in $P P 1$ methylation and reelin demethylation returned to baseline levels $24 \mathrm{~h}$ after conditioning, suggesting dynamic regulation of both DNA methylation and demethylation in the rat hippocampus. Likewise, DNMT inhibitors impair the induction of LTP at hippocampal synapses, providing an important cellular correlate of learning deficits induced by blocking DNA methylation [67]. Interestingly, DNMT inhibition in the prefrontal cortex impairs the recall of existing memories, but not the formation of new memories, indicating circuit-specific roles for DNA methylation in memory formation and maintenance [65 • ]. These data suggest that during memory formation, several plasticity permissive genes undergo decreased methylation and increased expression, and DNMT inhibitors were found to impair memory formation.

\section{Epigenetics and Aging}

Aging has been defined as a process of cellular senescence of adult tissues that results in compromised stress response, greater homeostatic imbalance and elevated risk of diseases [68]. Individual cells, which are genetically identical, can have substantially different responses to outside stimuli and markedly different lifespans, indicating that epigenetic factors, like DNA methylation, histone modifications and microRNAs, play an important role in gene expression and aging along with genetic factors $[63,69,70 \bullet]$.

The relationship between aging and epigenetics was reported nearly half a century ago, describing a global reduction of genomic DNA methylation with age [71]. Later, a global loss of cytosine methylation during aging was shown in the rat brain and heart [72]. Fuke and colleagues found an age-dependent decrease in global methylation levels in human leukocytes [73]. Several reports showed that global DNA hypomethylation appears to be an important feature of aging cells and tissues and is observed in many different age-related diseases, such as cancer, atherosclerosis, Alzheimer's and other neurodegenerative and autoimmune diseases [74, 75]. Distinct from the global decrease in DNA methylation, methylation levels can be either increased or decreased depending on the specific gene. For example, $c$-fos is methylated during aging in the liver, but not in the brain or spleen [76], suggesting that DNA hypermethylation is also important in aging. Therefore, two specific alterations of DNA methylation occur during aging: global DNA hypomethylation and aberrant promoter hypermethylation. It has been hypothesized that the accumulation of epigenetic alterations during aging may alter the risk of complex diseases, especially contributing to malignant transformation [77].

Other epigenetic mechanisms, like histone modifications, are also known to change during aging. For example, recent findings demonstrated that memory disturbances in the aging brain of the mouse were associated with the lack of a specific histone, H4 lysine 12 acetylation, which can be rescued by treatment with an HDAC inhibitor to restore memory function [78•]. Another example comes from silent information regulator 2 (Sir2, from the sirtuin family), which has HDAC activity and is associated with aging and energy-related processes. Sirtuins are a family of nicotinamide adenine dinucleotide-dependent HDACs, that are involved in a range of cellular events, including chromatin remodelling, transcriptional silencing, mitosis and the control of lifespan [79]. Sir2 was initially described in yeast, in which its deletion shortened replicative lifespan, whereas an extra copy of the gene increased lifespan, suggesting that the Sir2 family has an important role in aging. In mammals, the Sirtuin family has seven members (SIRT1-7), which are able to remove the acetyl group not only from histones but also 
from a varied range of substrates, and that are involved in the regulation of gene expression, stress responses, DNA repair, apoptosis, cell cycle, genomic stability and insulin regulation [80]. Mammalian SIRT1 (which features a deacetylase activity similar to that observed in yeast Sir2) can act on key transcription factors, such as tumor protein p53, forkhead transcriptional factors, p300 histone acetyltransferase, the tumor protein p73 and others, therefore they may modulate age-related diseases (cancer, metabolic, cardiovascular, neurodegenerative diseases) and various biological processes like cell survival under stress and cellular senescence [77].

There is evidence that microRNAs might also contribute to aging. The first evidence about microRNAs as epigenetic regulators of aging came from studies in Caenorhabditis elegants, where the microRNA lin-4 extended lifespan in the worm [81]. By contrast, other microRNAs like let-7 and miR-1 modulate the age-related decline. The microRNAs expression array in the livers of mice aged between four and 33-months-old showed that during aging there are more upregulated than downregulated microRNAs [81]. The list of microRNAs associated with mammalian aging is rapidly increasing. The data indicate that microRNAs represent an unexplored and promising field of aging epigenetics and further studies are need to clarify the molecular mechanisms that characterize the aging process.

\section{Epigenetic Mechanisms in Drug Addiction}

Drug and alcohol dependence are common, debilitating psychiatric disorders that are associated with high morbidity and mortality rates [82]. One common mechanism of recreational drugs is thought to be activation of the brain's reward circuitry, which centers on dopaminergic neurons in the ventral tegmental area of the midbrain and their projections to the limbic system - in particular, the nucleus accumbens (NAc, also known as the ventral striatum), dorsal striatum, amygdala, hippocampus and regions of the prefrontal cortex [83]. These changes occur alongside equally long-lasting changes in expression of genes such as $\Delta$ Fos $B, B d n f$, and $C r e b$ in the striatum [84•, 85]. Therefore, drug-induced neuronal plasticity mediated via alterations in gene expression has long been viewed as a major molecular mechanism for the development of drug addiction and relapse [86, 87].

Like other common complex diseases, addiction is a multifactorial and polygenic disorder that does not conform to a simple Mendelian transmission pattern [82]. Individuals are differentially vulnerable to substance abuse; not everyone who uses an addictive substance becomes addicted, only a subset of users experience the loss of control over drug use and compulsion for drug seeking and taking that defines the addicted state. Extensive epidemiological studies show that roughly half of an individual's risk for drug addiction is genetic, but the specific genes that confer risk for drug addiction remain largely unknown [82, 83, 87]. Entrance into an addicted state clearly results from the interplay between inherited predisposition (e.g. via genetic variants mediating the personality traits associated with drug seeking behavior and dependence) and the environment (e.g. actual exposure to drugs of abuse) [82, 87, 88]. For example, an approximately fivefold increase in genetic influence on alcohol consumption is observed in urban environments where there are a greater number of youths and higher alcohol sales compared to rural settings [89]. The addictive phenotype can persist for the length of an individual's life with drug craving and relapse occurring after weeks, months or even years of abstinence, and this persistence suggests that drugs induce long-lasting changes in the brain that underlie addictive behaviors [83, 87]. Thus, understanding the mechanisms that predispose individuals to the environmental factors associated with drug-taking behavior and the systems that translate the response to environmental stimuli (e.g. drug exposure) into longlasting cellular memories in the brain are fundamental to unlocking the neurobiological changes believed to play a role in addiction.

Multiple drugs of abuse (e.g. cocaine, amphetamine, ethanol) induce changes in histone modifications in the brain, and evidence has begun to accumulate that these modifications underlie some of the functional abnormalities found in addiction models [84•, 90, 91]. For example, at global levels of histone, $\mathrm{H} 3$ and $\mathrm{H} 4$ acetylation are increased in the rodent NAc after acute or chronic exposure to cocaine [84•]. However, at gene level, acute cocaine treatment has demonstrated a relation with histone $\mathrm{H} 4$ hyperacetylation at the promoters of the immediate early genes like $c$-Fos and FosB, while repeated cocaine treatment was associated with histone H3 hyperacetylation at the promoters of $C d k 5$ and $B d n f$. Further experiments have demonstrated that modifications of HDAC activity are important regulators of the rewarding properties of cocaine. Short-term systemic or intra-NAc administration of nonspecific HDAC inhibitors prior to cocaine or morphine exposure enhances behavioral preferences for places associated with drug delivery (socalled conditioned place preference) $[84 \bullet, 92]$. Studies of specific HDAC isoforms have yielded interesting information: overexpression of HDAC4 or HDAC5 decreases behavioral responses to cocaine [84•, 93•], whereas genetic deletion of HDAC5 hypersensitizes mice to the chronic effects (but not to the acute effects) of the drug [93•].

Histone phosphorylation is also an important component of the epigenetic response to drugs of abuse. Brami-Cherrier and colleagues reported that cocaine induces a robust phosphorylation of histone $\mathrm{H} 3$ within the NAc at the promoters 
of c-Fos and c-Jun [28] and $\mathrm{H} 3$ phosphorylation is positively regulated by MAPK/extracellular-signal regulated kinase (ERK) cascade, including phosphorylation of ERK and MSK-1-induced phosphorylation of histone H3 [94]. Nuclear accumulation of $32 \mathrm{kDa}$ dopamine and cAMP-regulated phosphoprotein (DARPP-32) acts to inhibit PP1, thereby preventing histone dephosphorylation [95]. These pathways control behavioral responses to cocaine through the inhibition of dopamine D1 receptors, ERK, DARPP-32 and MSK1 , all of which diminish drug-induced locomotor responses or drug conditioned place preference [28, 95]. Thus, these data confirm that changes in histone modifications may influence the transcription of genes involved in mediating cocaine-induced behavior.

Although the majority of studies have focused on histone modifications, DNA methylation is also a critical component of the epigenetic response to drug-related behaviors. Several recent studies have provided crucial evidence for the role of DNA methylation in cocaine-induced neuronal plasticity in the NAc and hippocampus [96, 97••]. Reports demonstrated that acute cocaine treatment induces rapid changes in the expression of DNMT3A and DNMT3B genes in the NAc, suggesting dynamic control of DNA methylation by drugs of abuse. Cocaine treatment also resulted in increased methylation of PP1 promoter region and binding of methyl $\mathrm{CpG}$ binding protein 2 (MeCP2) at promoter [96]. These changes are associated with transcriptional downregulation of PPIc in NAc. In contrast, acute and repeated cocaine administrations induced hypomethylation and decreased binding of MeCP2 at the FosB promoter, and these are associated with transcriptional upregulation of Fos $B$ in NAc [96]. Im and colleagues investigated a possible role of $\mathrm{MeCP} 2$ in the dorsal striatum in the escalating cocaine intake seen in rats with extended access to the drug, and found that MeCP2 knockdown prevents escalation of cocaine self-administration during extended access [98••]. Importantly, NAc-specific manipulations that block DNA methylation were shown to potentiate cocaine reward, whereas NAc-specific DNMT3A overexpression attenuated cocaine reward [97••]. Recent reports indicate that epigenetic changes in brain regions outside of the striatum are also important regulators of drug memories. For example, it has been shown that DNA methylation within the hippocampus and prelimbic cortex is necessary for the establishment and maintenance of cocaine conditioned place preference [99]. Taken together, these studies provide evidence that epigenetic modifications, including both DNA methylation and histone modifications, play an essential regulatory role in drug-induced synaptic plasticity and long-term behavioral adaptation in the nervous system. Evidence from these studies not only demonstrate the imperative role of epigenetic modifications in regulating behavioral responses to drug exposure, but also shed light on the complex mechanism of drug addiction. However, it is worth noting that only a few epigenetic studies on addiction have been performed in humans. Further studies are needed to shed light on the possible role of epigenetic mechanisms in individual's vulnerability to substance abuse.

\section{Conclusions}

In this paper, we have introduced the importance of epigenetic processes in regulating gene expression and described the role that dynamic epigenetic changes may play in memory mechanisms, aging, and complex diseases such as drug abuse via long-lasting transcriptional changes following repeated drug exposure. Accumulating evidence suggests that epigenetic processes may be the underlying mechanisms via the environmental factor, altering gene expression and therefore changing the risk of the complex diseases. Because epigenetic mechanisms are dynamic and reversible, chemical agents that alter the modification of histones or the methylation of DNA might prove to be potent candidates for therapeutic interventions. Moreover, the identification of specific epigenetic patterns associated with specific disease phenotypes might be useful biomarkers for early disease diagnosis and preventive intervention.

Disclosure No potential conflicts of interest relevant to this article were reported.

\section{References}

Papers of particular interest, published recently, have been highlighted as:

- Of importance

•• Of major importance

1. Haig D. The (dual) origin of epigenetics. Cold Spring Harb Symp Quant Biol. 2004;69:67-70.

2. McQuown SC, Wood MA. Epigenetic regulation in substance use disorders. Curr Psychiatry Rep. 2010;12:145-53.

3. DeAngelis JT, Farrington WJ, Tollefsbol TO. An overview of epigenetic assays. Mol Biotechnol. 2008;38:179-83.

4. Waterland RA. Epigenetic mechanisms and gastrointestinal development. J Pediatr. 2006;149:S137-142.

5. Gonzalo S. Epigenetic alterations in aging. J Appl Physiol. 2010;109:586-97.

6. - Strahl BD, Allis CD. The language of covalent histone modifications. Nature. 2000;403:41-5. This study provides the first demonstration that distinct histone modifications, on one or more tails, act sequentially or in combination to form a 'histone code' that is, read by other proteins to bring about distinct downstream events.

7. Grunstein M. Histone acetylation in chromatin structure and transcription. Nature. 1997;389:349-52. 
8. Felsenfeld G, Groudine M. Controlling the double helix. Nature. 2003;421:448-53.

9. Li B, Carey M, Workman JL. The role of chromatin during transcription. Cell. 2007;128:707-19.

10. Berger SL. The complex language of chromatin regulation during transcription. Nature. 2007;447:407-12.

11. Allis CD, Berger SL, Cote J, et al. New nomenclature for chromatin-modifying enzymes. Cell. 2007;131:633-6.

12. Berger SL, Kouzarides T, Shiekhattar R, et al. An operational definition of epigenetics. Genes Dev. 2009;23:781-3.

13. Kouzarides T. Chromatin modifications and their function. Cell. 2007;128:693-705.

14. Gibney ER, Nolan CM. Epigenetics and gene expression. Heredity (Edinb). 2010;105:4-13.

15. Norton VG, Imai BS, Yau P, et al. Histone acetylation reduces nucleosome core particle linking number change. Cell. 1989;57:449-57.

16. Barrett RM, Wood MA. Beyond transcription factors: the role of chromatin modifying enzymes in regulating transcription required for memory. Learn Mem. 2008;15:460-7.

17. Kuo MH, Allis CD. Roles of histone acetyltransferases and deacetylases in gene regulation. Bioessays. 1998;20:615-26.

18. Jenuwein T, Allis CD. Translating the histone code. Science. 2001;293:1074-80.

19. Narlikar GJ, Fan HY, Kingston RE. Cooperation between complexes that regulate chromatin structure and transcription. Cell. 2002;108:475-87.

20. Borrelli E, Nestler EJ, Allis CD, et al. Decoding the epigenetic language of neuronal plasticity. Neuron. 2008;60:961-74.

21. Tachibana M, Sugimoto K, Fukushima T, et al. Set domaincontaining protein, G9a, is a novel lysine-preferring mammalian histone methyltransferase with hyperactivity and specific selectivity to lysines 9 and 27 of histone H3. J Biol Chem. 2001;276:25309-17.

22. Shi Y, Lan F, Matson C, et al. Histone demethylation mediated by the nuclear amine oxidase homolog LSD1. Cell. 2004;119:941-53.

23. Hake SB, Xiao A, Allis CD. Linking the epigenetic 'language' of covalent histone modifications to cancer. $\mathrm{Br} \mathrm{J}$ Cancer. 2004;90:761-9.

24. Shi Y, Whetstine JR. Dynamic regulation of histone lysine methylation by demethylases. Mol Cell. 2007;25:1-14.

25. Scharf AN, Imhof A. Every methyl counts-Epigenetic calculus. FEBS Lett. 2011;585:2001-7.

26. Maze I, Nestler EJ. The epigenetic landscape of addiction. Ann NY Acad Sci. 2011;1216:99-113.

27. Su IH, Tarakhovsky A. Lysine methylation and 'signaling memory'. Curr Opin Immunol. 2006;18:152-7.

28. Brami-Cherrier K, Roze E, Girault JA, et al. Role of the ERK/ MSK1 signalling pathway in chromatin remodelling and brain responses to drugs of abuse. J Neurochem. 2009;108:1323-35.

29. Koshibu K, Gräff J, Beullens M, et al. Protein phosphatase 1 regulates the histone code for long-term memory. J Neurosci. 2009;29:13079-89.

30. Grant PA. A tale of histone modifications. Gen Biol. 2001;2(4): reviews0003.1-0003.6.

31. Barski A, Cuddapah S, Cui K, et al. High-resolution profiling of histone methylations in the human genome. Cell. 2007;129:82337.

32. Wang Z, Zang C, Rosenfeld JA, et al. Combinatorial patterns of histone acetylations and methylations in the human genome. Nat Genet. 2008;40:897-903.

33. Turner BM. Histone acetylation and an epigenetic code. Bioessays. 2000;22:836-45.

34. Campos EI, Reinberg D. Histones: annotating chromatin. Annu Rev Genet. 2009;43:559-99.
35. Day JJ, Sweatt JD. Epigenetic mechanisms in cognition. Neuron. 2011;70:813-29.

36. Holliday R, Pugh JE. DNA modification mechanisms and gene activity during development. Science. 1975;187:226-32.

37. Klose RJ, Bird AP. Genomic DNA methylation: the mark and its mediators. Trends Biochem Sci. 2006;31:89-97.

38. Bird A. DNA methylation patterns and epigenetic memory. Genes Dev. 2002;16:6-21.

39. Chahrour M, Zoghbi HY. The story of Rett syndrome: from clinic to neurobiology. Neuron. 2007;56:422-37.

40. Robertson KD, Wolffe AP. DNA methylation in health and disease. Nat Rev Genet. 2000;1:11-9.

41. Kim JK, Samaranayake M, Pradhan S. Epigenetic mechanisms in mammals. Cell Mol Life Sci. 2009;66:596-612.

42. Yasui N, Nogi T, Kitao T, et al. Structure of a receptor-binding fragment of reelin and mutational analysis reveal a recognition mechanism similar to endocytic receptors. Proc Natl Acad Sci U S A. 2007;104:9988-93.

43. •- Chahrour M, Jung SY, Shaw C, et al. MeCP2, a key contributor to neurological disease, activates and represses transcription. Science. 2008;320:1224-9. Using MeCP2 overexpressed or knockout mouse models, the authors show that MeCP2 dysfunction induced changes in the expression levels of thousands of genes, but the majority of genes (approximately 85\%) appeared to be activated by MeCP2. Furthermore, MeCP2 associates with the transcriptional activator CREB1 at the promoter of an activated target but not a repressed target. The authors conclude that MeCP2 regulates the expression of a wide range of genes and that it can function as both an activator and a repressor of transcription.

44. Eden A, Gaudet F, Waghmare A, et al. Chromosomal instability and tumors promoted by DNA hypomethylation. Science. 2003;300:455.

45. Villar-Garea A, Fraga MF, Espada J, et al. Procaine is a DNAdemethylating agent with growth-inhibitory effects in human cancer cells. Cancer Res. 2003;63:4984-9.

46. Goll MG, Bestor TH. Eukaryotic cytosine methyltransferases. Annu Rev Biochem. 2005;74:481-514.

47. Bestor TH. The DNA, methyltransferases of mammals. Hum Mol Genet. 2000;9:2395-402.

48. Siedlecki P, Zielenkiewicz P. Mammalian DNA methyltransferases. Acta Biochim Pol. 2006;53:245-56.

49. Ooi SK, Bestor TH. The colorful history of active DNA demethylation. Cell. 2008;133:1145-8.

50. Kangaspeska S, Stride B, Metivier R, et al. Transient cyclical methylation of promoter DNA. Nature. 2008;452:112-5.

51. Métivier R, Huet G, Gallais R, et al. Dynamics of estrogen receptor-mediated transcriptional activation of responsive genes in vivo: apprehending transcription in four dimensions. Adv Exp Med Biol. 2008;617:129-38.

52. Bergmann A, Lane ME. Hidden targets of microRNAs for growth control. Trends Biochem Sci. 2003;28:461-3.

53. Taft RJ, Pang KC, Mercer TR, et al. Non-coding RNAs: regulators of disease. J Pathol. 2010;220:126-39.

54. Szyf M, McGowan P, Meaney MJ. The social environment and the epigenome. Environ Mol Mutagen. 2008;49:46-60.

55. Li MD, van der Vaart AD. MicroRNAs in addiction: adaptation's middlemen? Mol Psychiatry. 2011;16:1159-68.

56. Levenson JM, Sweatt JD. Epigenetic mechanisms in memory formation. Nat Rev Neurosci. 2005;6:108-18.

57. Chwang WB, O'Riordan KJ, Levenson JM, et al. ERK/MAPK regulates hippocampal histone phosphorylation following contextual fear conditioning. Learn Mem. 2006;13:322-8.

58. - Gupta S, Kim SY, Artis S, et al. Histone methylation regulates memory formation. J Neurosci. 2010;30:3589-99. This work implicated that histone methylation is required for proper long-term consolidation of contextual fear memories. 
59. Levenson JM, O'Riordan KJ, Brown KD, et al. Regulation of histone acetylation during memory formation in the hippocampus. J Biol Chem. 2004;279:40545-59.

60. Korzus E, Rosenfeld MG, Mayford M. CBP histone acetyltransferase activity is a critical component of memory consolidation. Neuron. 2004;42:961-72.

61. Vecsey CG, Hawk JD, Lattal KM, et al. Histone deacetylase inhibitors enhance memory and synaptic plasticity via CREB: CBP dependent transcriptional activation. J Neurosci. 2007;27:6128-40.

62. • Guan JS, Haggarty SJ, Giacometti E, et al. HDAC2 negatively regulates memory formation and synaptic plasticity. Nature. 2009;459:55-60. This article reports that neuron-specific overexpression of $H D A C 2$, but not that of $H D A C 1$, decreased dendritic spine density, synapse number, synaptic plasticity and memory formation. These observations encourage the development and testing of HDAC2-selective inhibitors for human diseases associated with memory impairment.

63. Penner MR, Roth TL, Barnes CA, et al. An epigenetic hypothesis of aging-related cognitive dysfunction. Front in Aging Neurosci. 2010;2:1-11.

64. Lubin FD, Roth TL, Sweatt JD. Epigenetic regulation of BDNF gene transcription in the consolidation of fear memory. J Neurosci. 2008;28:10576-86.

65. •• Miller CA, Gavin CF, White JA, et al. Cortical DNA methylation maintains remote memory. Nat Neurosci. 2010;13:664-6. This study provided novel evidence that persistent, gene-specific cortical hypermethylation was induced in rats by a single, hippocampus-dependent associative learning experience and pharmacologic inhibition of methylation 1 month after learning disrupted remote memory. The authors speculate that the adult brain utilizes DNA methylation to preserve long-lasting memories.

66. Miller CA, Sweatt JD. Covalent modification of DNA regulates memory formation. Neuron. 2007;53:857-69.

67. Levenson JM, Roth TL, Lubin FD, et al. Evidence that DNA (cytosine-5) methyltransferase regulates synaptic plasticity in the hippocampus. J Biol Chem. 2006;281:15763-73.

68. Rakyan VK, Down TA, Maslau S, et al. Human aging-associated DNA hypermethylation occurs preferentially at bivalent chromatin domains. Genome Res. 2010;20:434-9.

69. Mostoslavsky R, Chua KF, Lombard DB, et al. Genomic instability and aging-like phenotype in the absence of mammalian SIRT6. Cell. 2006;124:315-29.

70. • Oberdoerffer P, Michan S, McVay M, et al. SIRT1 redistribution on chromatin promotes genomic stability but alters gene expression during aging. Cell. 2008;135:907-18. Using embryonic stem cells, the authors show that mammalian SIRT1 represses repetitive $D N A$ and a functionally diverse set of genes across the mouse genome. In response to DNA damage, SIRT1 relocalizes to DNA breaks to promote repair, resulting in transcriptional changes that parallel those in the aging mouse brain. Increased SIRT1 expression promotes survival in a mouse model of genomic instability and suppresses age-dependent transcriptional changes.

71. Berdyshev GD, Korotaev GK, Bioarskikh GV, et al. Nucleotide composition of DNA and RNA from somatic tissues of humpback and its changes during spawning. Biokhimiia. 1967;32:988-93.

72. Vanyushin BF, Nemirovski LE, Klimenko VV, et al. The 5methylcytosine in DNA of rats. Gerontologia. 1973;19:138-52.

73. Fuke C, Shimabukuro M, Petronis A, et al. Age related changes in 5-methylcytosine content in human peripheral leukocytes and placentas: an HPLC-based study. Ann Hum Genet. 2004;68:196-204.

74. Agrawal A, Tay J, Yang GE, et al. Age-associated epigenetic modifications in human DNA increase its immunogenicity. Aging (Albany NY). 2010;2:93-100.

75. Wierda RJ, Geutskens SB, Jukema JW, et al. Epigenetics in atherosclerosis and inflammation. J Cell Mol Med. 2010;14:1225-40.
76. Liu L, Wylie RC, Andrews LG, et al. Aging, cancer and nutrition: the DNA methylation connection. Mech of Ageing and Develop. 2003;124:989-98.

77. Calvanese V, Lara E, Kahn A, et al. The role of epigenetics in aging and age-related diseases. Ageing Research Reviews. 2009;8:268-76.

78. - Peleg S, Sananbenesi F, Zovoilis A, et al. Altered histone acetylation is associated with agedependent memory impairment in mice. Science. 2010;328:753-6. This article reports that during learning, aged mice display a specific deregulation of histone H4 lysine 12 acetylation and fail to initiate a hippocampal gene expression program associated with memory consolidation. Restoration of physiological H4 lysine 12 acetylation reinstates the expression of learning-induced genes and leads to the recovery of cognitive abilities. The authors conclude that deregulated H4K12 acetylation may represent an early biomarker of an impaired genome-environment interaction in the aging mouse brain.

79. Guarente L, Picard F. Calorie restriction - the SIR2 connection. Cell. 2005; 120:473-82.

80. Finkel T, Deng CX, Mostoslavsky R. Recent progress in the biology and physiology of sirtuins. Nature. 2009;460:587-91.

81. Maes OC, An J, Sarojini H, et al. Murine microRNAs implicated in liver functions and aging process. Mech Ageing Dev. 2008;129:534-41.

82. Goldman D, Oroszi G, Ducci F. The genetics of addictions: uncovering the genes. Nat Rev Genet. 2005;6:521-32.

83. Hyman SE, Malenka RC, Nestler EJ. Neural mechanisms of addiction: the role of reward-related learning and memory. Annu Rev Neurosci. 2006;29:565-98.

84. - Kumar A, Choi KH, Renthal W, et al. Chromatin remodeling is a key mechanism underlying cocaine-induced plasticity in striatum. Neuron. 2005;48:303-14. This study provides the first demonstration that acute cocaine administration induced transient $H 4$ hyperacetylation of various genes, e.g. cFos and FosB. Chronic cocaine injection did not have an effect on the c-fos promoter, but resulted in $\mathrm{H} 3$ hyperacetylation of the FosB promoter, as well as the Cdk5 and Bdnf genes. These histone modifications were shown to be long-lasting, persisting for at least 1 week after the last cocaine injection.

85. McClung CA, Nestler EJ. Regulation of gene expression and cocaine reward by CREB and DeltaFosB. Nat Neurosci. 2003;6:1208-15.

86. Kalivas PW, O'Brien C. Drug addiction as a pathology of staged neuroplasticity. Neuropsychopharmacology. 2008;33:166-80.

87. Nestler EJ. Molecular basis of long-term plasticity underlying addiction. Nat Rev Neurosci. 2001;2:119-28.

88. Wong CC, Mill J, Fernandes C. Drugs and addiction: an introduction to epigenetics. Addiction. 2011;106:480-9.

89. Dick DM, Rose RJ, Viken RJ, et al. Exploring gene-environment interactions: socioregional moderation of alcohol use. J Abnorm Psychol. 2001;110:625-32.

90. Kalda A, Heidmets LT, Shen HY, et al. Histone deacetylase inhibitors modulates the induction and expression of amphetamine-induced behavioral sensitization partially through an associated learning of the environment in mice. Behav Brain Res. 2007;181:76-84.

91. Kim JS, Shukla SD. Acute in vivo effect of ethanol (binge drinking) on histone $\mathrm{H} 3$ modifications in rat tissues. Alcohol Alcohol. 2006; 41:126-32.

92. Sanchis-Segura C, Lopez-Atalaya JP, Barco A. Selective boosting of transcriptional and behavioral responses to drugs of abuse by histone deacetylase inhibition. Neuropsychopharmacology. 2009;34:2642-54.

93. - Renthal W, Maze I, Krishnan V, et al. Histone deacetylase 5 epigenetically controls behavioral adaptations to chronic emotional stimuli. Neuron. 2007;56:517-29. This study identifyied the activity-dependent histone deacetylase (HDAC5) as a central integrator of stress and drugs stimuli with changes in chromatin structure 
and gene expression. Chronic, but not acute, exposure to cocaine or stress decreases HDAC5 function in the nucleus accumbens (NAc), which allows for increased histone acetylation and transcription of HDAC5 target genes.

94. Bertran-Gonzalez J, Bosch C, Maroteaux M, et al. Opposing patterns of signaling activation in dopamine $\mathrm{D} 1$ and $\mathrm{D} 2$ receptorexpressing striatal neurons in response to cocaine and haloperidol. J Neurosci. 2008;28:5671-85.

95. Stipanovich A, Valjent E, Matamales M, et al. A phosphatase cascade by which rewarding stimuli control nucleosomal response. Nature. 2008;453:879-84.

96. Anier K, Malinovskaja K, Aonurm-Helm A, et al. DNA methylation regulates cocaine-induced behavioral sensitization in mice. Neuropsychopharmacology. 2010;35:2450-61.

97. •- LaPlant Q, Vialou V, Covington HE, et al. Dnmt3a regulates emotional behavior and spine plasticity in the nucleus accumbens. Nat Neurosci. 2010;13:1137-43. This study provided crucial evidence of the importance of DNMT3A in the $N A c$ in regulating cellular and behavioral plasticity to repeated cocaine exposure.

98. •- Im HI, Hollander JA, Bali P, et al. MeCP2 controls BDNF expression and cocaine intake through homeostatic interactions with microRNA-212. Nat Neurosci. 2010;13:1120-7. This work provided an alternative mechanism for MeCP2 action in addiction models. The authors showed that MeCP2 controls cocaine intake through microRNA-mediated regulation of BDNF, demonstrating the complex interactions among the various mechanisms of epigenetic modifications in the drug-exposed brain.

99. Han J, Li Y, Wang D, et al. Effect of 5-aza-2-deoxycytidine microinjecting into hippocampus and prelimbic cortex on acquisition and retrieval of cocaine-induced place preference in $\mathrm{C} 57 \mathrm{BL} / 6$ mice. Eur J Pharmacol. 2010;642:93-8. 